\title{
Programación temprana de la hipertensión arterial
}

\author{
Early programming of hypertension
}

\author{
Adriana Iturzaeta ${ }^{a}$ (D), María M. Sáenz Tejeira ${ }^{b}$ (D)
}

\section{RESUMEN}

La hipertensión arterial (HTA) es un factor de riesgo modificable deenfermedad cardiovascular (ECV) y debe incluirse dentro del estudio de los orígenes del desarrollo de la salud y enfermedad (DOHaD).

Durante el desarrollo intrauterino y perinatal, diferentes factores ambientales impactan en la programación temprana de las enfermedades crónicas no transmisibles (ECNT).

En esta revisión se resume la evidencia que vincula los cambios adaptativos y la plasticidad del feto a factores ambientales desfavorables alterando el fenotipo adulto en el desarrollo de HTA. Estos cambios adaptativos responden a cambios epigenéticos que favorecen el desarrollo deHTA y ECV en la edad adulta con implicancias intergeneracionales.

Por último, se mencionan estrategias preventivas para limitar o revertir algunas de las variables que pueden producir alteraciones en la programación del desarrollo que conducen a HTA en etapas más tardías de la vida.

Palabras clave: presión arterial, hipertensión, programación fetal, $\mathrm{DOHaD}$, epigenética.

https://decs.bvsalud.org/es/

Sociedad Argentina de Pediatría.

b. Hospital Italiano de Buenos Aires, Ciudad Autónoma de Buenos Aires, Argentina; Hospital General de Agudos Dr. Juan A. Fernández, Ciudad Autónoma de Buenos Aires, Argentina. Comité de Cardiología, Sociedad Argentina de Pediatría.

Correspondencia: Adriana Iturzaeta: aiturzaeta@yahoo.com. ar

Financiamiento:

Ninguno.

Conflicto de intereses: Ninguno que declarar.

Recibido: 17-3-2021

Aceptado: 21-7-2021 http: / / dx.doi.org/10.5546/ aap.2022.e8

Texto completo en inglés:

http: / / dx.doi.org/10.5546/ aap.2022.eng.e8

Cómo citar: Iturzaeta A, Sáenz Tejeira MM.

Programación temprana de la hipertensión arterial. Arch Argent Pediatr 2022;120(1):e8-e16.

\section{INTRODUCCIÓN}

La hipertensión arterial (HTA) es un importante factor de riesgo modificable de desarrollo de enfermedad cardiovascular (ECV) en el adulto que tiene su origen en la infancia y su prevalencia se estima un $3,5-5 \%$ en la población pediátrica. ${ }^{1-5}$
Dado el gran impacto que representa la ECV en el adulto a nivel de salud pública, surgieron estrategias para identificar factores de riesgo que reduzcan la morbimortalidad de la población..$^{3-5}$

En tal sentido, la incorporación del concepto de orígenes del desarrollo de la salud y la enfermedad ( $\mathrm{DOHaD})$ le otorga un papel fundamental como factor desencadenante de las enfermedades crónicas no transmisibles (ECNT) a la alteración del desarrollo normal desde antes de la concepción, a la vida intrauterina, a la perinatal y al entorno de la vida temprana. Ciertos factores ambientales y el entorno materno adverso durante la gestación y la vida perinatal pueden influir en la plasticidad del desarrollo, en el cual el feto se adapta a las condiciones ambientales con riesgo futuro de desarrollar HTA y ECV durante la edad adulta, así como otras enfermedades metabólicas, del neurodesarrollo e inmunológicas. ${ }^{3-5}$

La evidencia también demuestra que los efectos de la programación ante la exposición materna y paterna a los desafíos ambientales pueden transmitirse a través de generaciones. ${ }^{5}$

Por tal motivo, es importante conocer y detectar los efectos de condiciones ambientales adversas en el desarrollo fetal y perinatal, los mecanismos epigenéticos y otras respuestas adaptativas que pueden ser centrales en la programación temprana de la HTA en la vida futura, y evaluar estrategias destinadas a reducir el riesgo. ${ }^{5}$

\section{INFLUENCIAS AMBIENTALES EN EL DESARROLLO DE HTA}

Estudios epidemiológicos realizados en los últimos años 
demostraron que las etapas previas a la concepción, y los periodos fetal y perinatal pueden contribuir al desarrollo de HTA y de otros factores de riesgo de ECV en la edad futura (Tabla 1). . $^{3,4,6-10}$

Esta hipótesis, inicialmente propuesta por Barker, postula que el bajo peso al nacer (BPN) inducido por la desnutrición gestacional origina adaptaciones en el feto que predisponen o "programan" el desarrollo de HTA y ECV en etapas posteriores de la vida, proceso llamado de "programación fetal", que dio lugar posteriormente al concepto $\mathrm{DOHaD} .{ }^{10,11}$

El BPN, la prematuridad y el retraso del crecimiento intrauterino (RCIU) suelen ser el resultado de la plasticidad del desarrollo a factores adversos entre los que se destacan la falta de nutrientes, ya sea por inadecuada alimentación de la madre -como lo demuestran los estudios en la descendencia de poblaciones sometidas a hambrunas durante la etapa gestacional- o por una insuficiencia placentaria., ${ }^{3,4,9,12,13}$

Existe evidencia de que el BPN, la prematuridad, el efecto del rápido crecimiento posnatal en niños con bajo peso (cohorte de Helsinki y el Estudio Nord-Trondelag) y los cambios del índice de masa corporal (IMC) durante la infancia se asocian con riesgo de HTA posterior. ${ }^{3,4,13-18}$

Otro estudio coincide en que el antecedente del nacimiento prematuro fue el evento de mayor asociación con la presión arterial (PA) en la vida adulta; reportó un incremento significativo de PA sistólica (PAS) de 4,2 $\mathrm{mmHg}$, un aumento en la PA diastólica de $2,6 \mathrm{mmHg}$, un aumento de la PAS ambulatoria de 24 horas de $3,1 \mathrm{mmHg}$, y que las mujeres parecen tener mayor riesgo. ${ }^{19}$

La evidencia demuestra que existen períodos críticos en el desarrollo fetal (organogénesis) y en el período posnatal temprano, considerados los más sensibles a déficits nutricionales con riesgo de desarrollo futuro de HTA. ${ }^{13,20}$

Es importante destacar que no solo el BPN sino también el crecimiento fetal excesivo (macrosomía) predisponen a los individuos a tener mayor riesgo de desarrollar ECV en la vida adulta. Esto ocurre en los hijos de gestantes con

TABLA 1. Factores ambientales adversos y orígenes del desarrollo de la salud y la enfermedad ${ }^{6}$

\begin{tabular}{|c|c|c|c|}
\hline Etapa del desarrollo & Tipo de exposición & $\begin{array}{l}\text { Efectos en modelos } \\
\text { experimentales }\end{array}$ & $\begin{array}{l}\text { Observaciones } \\
\text { en humanos }\end{array}$ \\
\hline Preimplantación & $\begin{array}{l}\text { - Desnutrición o dieta con } \\
\text { bajo contenido de proteínas }\end{array}$ & $\begin{array}{l}\text { - Deficiencias celulares } \\
\text { en blastocistos }\end{array}$ & \\
\hline Placentaria & $\begin{array}{l}\text { - Insuficiencia placentaria } \\
\text { - Embarazos múltiples }\end{array}$ & $\begin{array}{l}\text { - Modelos de insuficiencia } \\
\text { placentaria asociada con RCIU }\end{array}$ & $\begin{array}{l}\text { - Tamaño placentario correlacionado } \\
\text { con el peso al nacer y con } \\
\text { enfermedades cardiovasculares } \\
\text { futuras en los estudios iniciales } \\
\text { de Barker }\end{array}$ \\
\hline \multirow[t]{2}{*}{ Intrauterina } & $\begin{array}{l}\text { - Hormonas (folato, } \\
\text { glucocorticoides) } \\
\text { - Compuestos metilados }\end{array}$ & $\begin{array}{l}\text { - Prueba de tolerancia a la } \\
\text { glucosa alterada, diabetes mellitus, } \\
\text { reducción de la masa de células } \beta \\
\text { - Obesidad, nefrogénesis } \\
\text { deteriorada }\end{array}$ & $\begin{array}{l}\text { - Prueba de tolerancia a la glucosa } \\
\text { alterada, diabetes mellitus, } \\
\text { reducción de la masa de células } \beta \\
\text { - Obesidad, nefrogénesis } \\
\text { deteriorada }\end{array}$ \\
\hline & $\begin{array}{l}\text { - Desnutrición } \\
\text { calórico-proteica } \\
\text { - Hiperglucemia } \\
\text { - Exceso de glucocorticoides } \\
\text { - Alteraciones del sistema } \\
\text { renina-angiotensina } \\
\text { - Inflamación } \\
\text { - Ácidos grasos libres }\end{array}$ & $\begin{array}{l}\text { - Aumento de la expresión del } \\
\text { receptor de angiotensina II } \\
\text { de tipo } 1 . \\
\text { - Anomalías cardiorrenales, } \\
\text { - Cambios estructurales } \\
\text { vasculares } \\
\text { - Hipertensión }\end{array}$ & $\begin{array}{l}\text { - Bajo número de nefronas } \\
\text { - Cambios estructurales vasculares } \\
\text { - Hipertensión }\end{array}$ \\
\hline Perinatal & $\begin{array}{l}\text { - Desnutrición } \\
\text { - Sobrenutrición } \\
\text { - Sustancias tóxicas } \\
\text { - Hipoxia } \\
\text { - Estrés oxidativo } \\
\text { - Deficiencias de metales }\end{array}$ & $\begin{array}{l}\text { - Cambios epigenéticos } \\
\text { - Síndrome cardiorrenal futuro } \\
\text { - Síndrome metabólico futuro }\end{array}$ & $\begin{array}{l}\text { - ECV futura } \\
\text { - Síndrome metabólico futuro }\end{array}$ \\
\hline
\end{tabular}

RCIU: retraso del crecimiento intrauterino. ECV: enfermedad cardiovascular. 
aumento del IMC corporal o diabéticas. El estado metabólico materno, la obesidad pregestacional y la ganancia excesiva de peso durante el embarazo estarían implicados en el círculo vicioso transgeneracional de la programación temprana de la PA. ${ }^{21}$

En estudios de cohorte en humanos, tanto la HTA durante el embarazo como la preeclampsia de inicio temprano se asociaron con el desarrollo de HTA en la descendencia, especialmente en el período neonatal, demostrando el rol de la insuficiencia placentaria en la programación de la PA.22

Además, existen otras causas de estrés fetal que provocan un inadecuado crecimiento fetal y se correlacionan con una mayor prevalencia de HTA en la adolescencia y en la edad adulta. Entre ellos cabe destacar la hipoxia, el estrés materno (ambiental, emocional y fisiológico), cambios en la microbiota, la exposición temprana a glucocorticoides, la exposición a tóxicos como la nicotina y el humo del tabaco, el consumo de alcohol y la privación de sueño materno. ${ }^{23-36}$

Los cambios en la microbiota pueden influir en la PA regulando la sensibilidad a la sal. La exposición temprana a niveles elevados de glucocorticoides altera la autorregulación placentaria de la $11 \beta$-hidroxiesteroide deshidrogenasa de tipo 2 con menor actividad en el sexo masculino, elevando las concentraciones de cortisol en la circulación fetal. Esta adaptación puede asociarse a RCIU, alteraciones en los procesos de la nefrogénesis, elevados niveles de PA, modificaciones del metabolismo de las grasas, resistencia a la insulina y variaciones del eje hipotálamo-hipófisis-adrenal en la adolescencia y en la vida adulta. La exposición a tóxicos, como el tabaquismo materno (nicotina), o al humo del tabaco causa vasoconstricción y se reduce el flujo sanguíneo placentario con alteración del desarrollo vascular y menor tamaño renal con aumento de la PA en la primera infancia independientemente de su efecto sobre el peso al nacer. También la exposición fetal al alcohol y a la cocaína puede afectar a múltiples órganos e incrementa potencialmente el riesgo de enfermedad y disfunción orgánica en la edad adulta. La restricción del sueño gestacional puede aumentar el IMC y la PA de la descendencia por alteraciones del metabolismo de la glucosa, vías inflamatorias y predisponer al parto prematuro, con efectos más pronunciados en las niñas; y en modelos animales puede elevar la PA por modificaciones del desarrollo renal. ${ }^{23-36}$

\section{ADAPTACIONES EN LA PROGRAMACIÓN DE LA HTA}

Como resultado de la interacción del genoma con un medio intrauterino desfavorable, el feto va generando respuestas estructurales y funcionales que a corto plazo son adaptativas y promueven posibilidades de supervivencia, pero a largo plazo pueden tener consecuencias adversas. Se postulan cambios en el desarrollo cardiovascular, renal y del sistema nervioso autónomo relacionados con la regulación de la PA. 5,37

\section{Alteraciones renales}

Los fenotipos renales informados en la programación del desarrollo de la HTA incluyen riñones pequeños al nacer con una reducción en el número de nefronas (si el efecto adverso se presenta en el período de mayor plasticidad nefrogénica, antes de las 34 semanas de edad gestacional), alteraciones en la función renal, en el transporte de sodio, en el sistema reninaangiotensina-aldosterona (SRAA) y alteraciones de los nervios simpáticos renales que regulan la función renal. ${ }^{5}$

Los factores maternos como la restricción de nutrientes que limitan el número de nefronas junto con la pérdida de nefronas relacionada con la edad se asociaron con la programación de la HTA en la edad adulta. ${ }^{38}$

Brenner y Chertow, sugieren que el riesgo de desarrollar HTA esencial en la edad adulta aumenta como consecuencia de una alteración en el desarrollo renal que acompaña el RCIU. Esta hipótesis está avalada por estudios en humanos donde demuestran que un menor número de nefronas en individuos con $\mathrm{BPN}$, con una menor área de filtración glomerular, causa hiperfiltración glomerular compensatoria y eventualmente hipertrofia glomerular, lesión renal (microalbuminuria, proteinuria) y glomeruloesclerosis, y favorece el desarrollo de HTA. ${ }^{38-41}$

Otras observaciones sugieren una relación directa entre el peso al nacer y el número de nefronas, una relación inversa entre el peso al nacer y HTA en la edad adulta, y una relación inversa entre el número de nefronas y la PA, independientemente de si el número de nefronas se reduce congénitamente o en la vida posnatal. ${ }^{40,41}$

La programación del desarrollo de HTA demostró que la tasa de filtración glomerular se reduce después de la restricción calórica y proteica de la dieta durante el embarazo, 
como así también con la administración de glucocorticoides, alcohol y diabetes materna. ${ }^{5}$

En los modelos de HTA programada, se observó lesión tubulointersticial con alteraciones en las vías que regulan la fibrosis, la inflamación, el estrés oxidativo, y los cambios en la expresión de genes y / o proteínas de los canales de sodio, calcio y agua que pueden alterar la función tubular con afectación de la homeostasis del sodio. $^{5}$

En modelos experimentales animales, se planteó la hipótesis de que las primeras modificaciones en el microambiente de la corteza renal cambian el manejo del sodio, con un aumento de la sensibilidad a la sal. En algunos niños prematuros o con BPN, puede observarse este aumento de cifras de PA ante ingesta excesiva de sodio por alteraciones de los mecanismos de su regulación relacionados ya sea por la disminución del área de filtración glomerular asociada a la reducción del número de nefronas o por el aumento de la reabsorción tubular del sodio. ${ }^{4,5,42,43}$

Existen pocos datos sobre los nervios renales y su papel potencial en la HTA programada, aunque la inervación simpática juega un papel importante en la regulación de la PA y el transporte de sodio. ${ }^{44}$

\section{Alteraciones cardiovasculares}

Las alteraciones en la estructura y la función cardíaca y vascular pueden contribuir significativamente a la HTA y están presentes en los modelos de HTA programada. ${ }^{3,4,5,45}$

El fenotipo cardíaco se caracteriza por la reducción en el número de cardiomiocitos y/o un aumento en la hipertrofia cardíaca en la descendencia después de la restricción de proteínas maternas, de la deficiencia de vitamina $\mathrm{D}$ y la restricción de la función placentaria en los modelos experimentales de programación fetal que se asocian con BPN. También se observó hipertrofia cardíaca en la descendencia masculina, disfunción diastólica y sistólica en la descendencia masculina y femenina de 3 meses ante una dieta rica en grasas durante la gestación en animales. ${ }^{3,4,46-50}$

En niños con antecedente de estrés fetal con $\mathrm{BPN}$, se observó vasodilatación dependiente del endotelio más reducida, independientemente del IMC actual. ${ }^{51}$ Estos datos muestran que los niños nacidos con BPN tienen disfunción endotelial..$^{52}$

Estudios experimentales avalan la posibilidad de que el estrés fetal pueda afectar la vasculogénesis y produzca disfunción endotelial y remodelación vascular. Estos cambios fenotípicos y funcionales de las células musculares lisas vasculares se producen en respuesta a mediadores vasoconstrictores y vasodilatadores secretados por las células endoteliales. Este proceso se caracteriza por cambios en la estructura vascular como la alteración del grosor de la pared y del diámetro de la luz (se destaca el papel de las metaloproteinasas) y por cambios en las propiedades mecánicas vasculares, los cuales pueden desarrollar o ser precursores de HTA a lo largo de la vida. ${ }^{5,50-54}$

En situaciones de RCIU, se postuló alteración en la síntesis de elastina durante la etapa fetal, período de rápido crecimiento vascular, que podría conducir a cambios en la mecánica vascular, con disminución de la elasticidad arterial y al desarrollo de HTA a largo plazo. ${ }^{55}$

Esta hipótesis está avalada por la asociación entre el BPN y la rigidez arterial encontrada en niños prematuros ante mediciones de la velocidad de la onda de pulso elevadas, con significado clínico del envejecimiento vascular acelerado. ${ }^{56-57}$

\section{Alteraciones del sistema nervioso autónomo}

Existe fuerte evidencia de que el aumento de la actividad del sistema nervioso simpático contribuye a la patogénesis de la HTA esencial y puede originarse desde el inicio del desarrollo a través de la modulación de la reabsorción de sodio a lo largo de los túbulos renales. Esta hiperactividad simpática puede ser un mecanismo subyacente común en la HTA inducida por la obesidad materna. ${ }^{3,4,5,58}$

Además, hay interacciones bidireccionales entre el sistema nervioso simpático y el sistema inmune. En particular, la relación entre la inmunidad adaptativa de los linfocitos $\mathrm{T}$ y el sistema nervioso entérico, que a través de la composición de la microbiota aumenta las citoquinas proinflamatorias y altera la permeabilidad intestinal interviniendo en la programación de la HTA sal sensible. ${ }^{54,59,60}$

\section{MECANISMOS QUE CONTRIBUYEN A LA PROGRAMACIÓN DE LA HTA}

Las alteraciones orgánicas previamente mencionadas pueden estar asociadas con cambios en diversos mediadores entre los que destacan el SRAA, la modulación epigenética de algunos genes (particularmente los relacionados con el factor de crecimiento insulinoide [IGF]) y las especies reactivas de oxígeno (ROS). ${ }^{5}$

Todos los componentes del SRAA son 
afectados en la programación del desarrollo de la HTA en una variedad de modelos -que incluyen la administración de glucocorticoides, dieta con alto contenido de grasas, sodio, sacarosa, fructosa y baja en proteínas-, que permiten explicar las alteraciones orgánicas en la función renal y vascular. ${ }^{61-64}$

Los hallazgos comunes de estos modelos sugieren que la restricción proteica en la dieta materna conduce a la supresión del SRAA intrarrenal perinatal con posterior deterioro de la nefrogénesis y reducción del número de nefronas, y predispone a HTA en el adulto. También, hay diferencias en uno y otro sexo informadas en la programación de la PA durante la nefrogénesis., 763,65

Las modificaciones epigenéticas al principio de la vida embrionaria suponen cambios en la expresión génica, regulan su función, pero sin alterar la secuencia de ADN y estos cambios pueden transmitirse a las generaciones futuras. ${ }^{5,66}$

Estos mecanismos epigenéticos implican la metilación del ADN (mecanismo más estudiado en los modelos de programación de desarrollo de la HTA), la modificación de histonas, el control de la expresión de genes por ARN no codificantes y la conformación de cromatina. 5,67

Estudios experimentales en humanos y animales sugieren que los cambios epigenéticos son uno de los mecanismos responsables de la programación fetal que podrían explicar tanto las alteraciones orgánicas como la disfunción vascular y HTA en la descendencia. Estos cambios epigenéticos consisten en modificaciones en genes relacionados con el SRAA, el receptor de angiotensina 1 , el tono vascular, los canales iónicos, canales epiteliales de sodio, cotransportador de $\mathrm{Na}^{+}-\mathrm{K}^{+}-2 \mathrm{Cl}^{-}$, aumento de la expresión de micro-ARN que regula la traducción de la enzima convertidora de angiotensina 1, micro-ARN asociados a lesión cardíaca, angiogénesis y cambios celulares, modificaciones en las histonas de óxido nítrico sintasa endotelial (eNOS) e hipermetilación del HSD11B2 entre otros (Tabla 2). 3,4,68-70

Estudios en humanos que sufrieron restricción calórica y de nutrientes durante el período intrauterino demostraron modificaciones en la metilación del gen del IGF , en genes involucrados en la función cardiovascular y la inflamación. . $^{71,72}$

También hay que tener en cuenta que la metilación del ADN que ocurre en sitios de citosina-fosfato guanina está mediada por enzimas que dependen de niveles adecuados de micronutrientes, como colina, niacina, ácido fólico, vitamina $\mathrm{B}_{6} \mathrm{y} \mathrm{B}_{12}$, vitamina $\mathrm{C}$, metionina, glutatión, cinc, selenio y ácido retinoico. La deficiencia de estos puede generar en el feto menor oxigenación tisular, incremento del estrés oxidativo, activación de la apoptosis por disfunción mitocondrial, alteración en el desarrollo de órganos y puede predisponer al desarrollo del síndrome metabólico y a HTA en la vida adulta. . $^{31,54,73,74}$

También el entorno paterno, ante la restricción calórica antes y durante la pubertad, desempeña un papel en la programación de enfermedades metabólicas y cardiovasculares. ${ }^{75}$

Otro aporte importante de la biología molecular para tener en cuenta, que se relaciona con el origen fetal de las ECNT, es el acortamiento telomérico. Los telómeros son segmentos repetidos de $\mathrm{ADN}$ al final de los cromosomas, cuya función principal es la de conferirles

TABLA 2. Algunos factores involucrados en la programación temprana de la $H T A^{4}$

\begin{tabular}{|c|c|c|c|c|}
\hline $\begin{array}{l}\text { Regulación } \\
\text { epigenética }\end{array}$ & $\begin{array}{l}\text { Cambios } \\
\text { hormonales }\end{array}$ & Cambios ROS & $\begin{array}{c}\text { Cambios } \\
\text { vasculares/hemodinámicos }\end{array}$ & $\begin{array}{c}\text { Cambios } \\
\text { transportadores }\end{array}$ \\
\hline Metilación del ADN & SRAA & $\begin{array}{l}\text { Incremento del } \\
\text { superóxido }\end{array}$ & $\begin{array}{l}\text { Microvasculatura } \\
\text { atenuada }\end{array}$ & ATPasa $\mathrm{Na}^{+} / \mathrm{K}^{+}$ \\
\hline Modificación de histonas & $\begin{array}{l}\text { Eje hipotálamo- } \\
\text { hipófisis }\end{array}$ & $\begin{array}{l}\text { Activación de la } \\
\text { NADPH-oxidasa }\end{array}$ & $\begin{array}{c}\text { Cambio en la } \\
\text { densidad capilar }\end{array}$ & ATPasa $\mathrm{Na}^{+} / \mathrm{K}^{+}$ \\
\hline ARN no codificante & Péptidos natriuréticos & & & NKCC2 \\
\hline Conformación de cromatina & & & & $\begin{array}{l}\text { Cotransportador } \\
\text { de } \mathrm{Na}^{+}-\mathrm{Cl}^{-}\end{array}$ \\
\hline
\end{tabular}

HTA: hipertensión arterial; SRAA: sistema renina-angiotensina-aldosterona; ROS: especies reactivas de oxígeno; ATPasa $\mathrm{Na}+\mathrm{K}+$ : adenosina-trifosfatasa de sodio y potasio; NADPH: fosfato de dinucleótido de nicotinamida y adenina; NKCC2: cotransportador de sodio, potasio y cloro; cotransportador $\mathrm{Na}^{+}-\mathrm{Cl}$ : cotransportador de sodio y cloro. Las columnas de esta tabla, no están relacionadas entre sí. 
estabilidad estructural y regular el tiempo de vida de las estirpes celulares. Su acortamiento, ante eventos adversos en la vida intrauterina, se asocia con el desarrollo de enfermedades crónicas, cardiovasculares, metabólicas y con la disminución de la esperanza de vida. ${ }^{76}$

Con respecto al estrés oxidativo, se postuló que es otro de los mediadores más plausibles entre un crecimiento fetal adverso y un aumento del riesgo de desarrollar ECV y metabólicas en la edad adulta. ${ }^{77}$

El exceso de especies reactivas del oxígeno (ROS, por su sigla en inglés) producido por las células vasculares, inmunes, dendríticas y sistemas enzimáticos podría explicar varias de las alteraciones orgánicas, como la disfunción endotelial con aumento del tono vascular, demostrada en modelos animales sometidos a restricción proteica durante la gestación. 8,54,78

Existe evidencia de que la desnutrición materna, la sobrecarga de sodio en el embarazo y la disfunción placentaria están asociadas con un aumento del estrés oxidativo y con el desequilibrio óxido nítrico-ROS en el riñón fetal, capaz de programar HTA en el adulto y enfermedad renal. ${ }^{54,79}$

\section{ESTUDIOS DE MODELOS DE EXPERIMENTACIÓN DE PROGRAMACIÓN PERINATAL DE LA PRESIÓN ARTERIAL}

Los estudios de modelos de experimentación sobre los mecanismos de la programación fetal para el desarrollo de HTA y ECV en la edad adulta posibilitan una mejor interpretación de los resultados obtenidos en humanos y permiten realizar estudios a largo plazo. Los más representativos son el modelo de restricción nutricional durante la gestación, de insuficiencia placentaria, el de hipoxia y el de exposición a glucocorticoides (Tabla 3). ${ }^{2,4,6-8,23}$

Estos modelos establecen que, entre los factores de riesgo cardiovascular, la HTA es el factor que se asocia con mayor frecuencia a RCIU debido a desequilibrios nutricionales. ${ }^{3,4}$

\section{MEDIDAS DE PREVENCIÓN EN POBLACIÓN DE RIESGO DE DESARROLLO DE HTA}

La evidencia actual sugiere que numerosos mecanismos participan en el desarrollo de HTA programada por un ambiente prenatal, perinatal y posnatal desfavorable..$^{3-5}$

Las estrategias clínicas de prevención para evitar el riesgo de HTA desde períodos críticos del desarrollo sugieren controlar y detectar factores de riesgo. Es importante evaluar antes y durante el embarazo la presencia de antecedentes infecciosos, consumo de medicamentos, alcohol, drogas o tabaco, realizar controles clínicos y ecográficos. Se deben registrar el peso, la talla, la PA y el estado metabólico, y controlar la ingesta de diferentes micronutrientes o minerales de la dieta. ${ }^{3,4,6,7,50,80,81}$

En los recién nacidos de BPN, prematuros, macrosómicos y con antecedentes familiares de HTA, realizar seguimiento clínico durante la infancia y adolescencia con controles de PA y glucemia, asegurar la lactancia materna y alimentación complementaria, implementar medidas de estilo de vida saludable y actividad

TABLA 3. Ejemplos de modelos de experimentación de programación temprana de HTA

\begin{tabular}{|c|c|c|c|c|}
\hline Modelo experimental & Especies estudiadas & Número de nefronas & Histología glomerular & Presión arterial \\
\hline Restricción calórica & Ratas, ratones, cobayas & Disminuido & Hipertrofia glomerular & $\begin{array}{l}\text { Normal o } \\
\text { aumentada }\end{array}$ \\
\hline $\begin{array}{l}\text { Dieta con bajo } \\
\text { contenido de } \\
\text { proteínas }\end{array}$ & $\begin{array}{l}\text { Ratas, ratones, } \\
\text { cobayas, ovejas }\end{array}$ & $\begin{array}{l}\text { Disminuido } \\
\text { pero variable }\end{array}$ & Hipertrofia glomerular & $\begin{array}{l}\text { Normal o } \\
\text { aumentada }\end{array}$ \\
\hline Dieta multideficiente & Ratas & Disminuido & Hipertrofia glomerular & $\begin{array}{l}\text { Normal o } \\
\text { aumentada }\end{array}$ \\
\hline Dexametasona & $\begin{array}{l}\text { Ratas, ratones, } \\
\text { cobayas, ovejas }\end{array}$ & $\begin{array}{l}\text { Disminuido } \\
\text { o sin cambios }\end{array}$ & $\begin{array}{l}\text { Hipertrofia glomerular o } \\
\text { glomeruloesclerosis segmentaria }\end{array}$ & $\begin{array}{l}\text { Normal o } \\
\text { aumentada }\end{array}$ \\
\hline Diabetes & Ratones, ratas & $\begin{array}{l}\text { Disminuido o } \\
\text { sin cambios }\end{array}$ & Hipertrofia glomerular & $\begin{array}{l}\text { Normal o } \\
\text { aumentada }\end{array}$ \\
\hline $\begin{array}{l}\text { Ligadura parcial } \\
\text { de la arteria uterina }\end{array}$ & Ratas & Disminuido & Hipertrofia glomerular & $\begin{array}{l}\text { Normal o } \\
\text { aumentada }\end{array}$ \\
\hline
\end{tabular}


física que permitan disminuir la posibilidad de desarrollar obesidad, síndrome metabólico, diabetes e HTA. ${ }^{1-4,6,7,50,80,81}$

\section{CONCLUSIÓN}

La acumulación de factores adversos durante la preconcepción, el crecimiento y desarrollo intrauterino, así como durante los primeros años de vida produce cambios epigenéticos que favorecen el desarrollo de HTA y ECV en la edad adulta con implicancias intergeneracionales. Estos factores adversos pueden ser reversibles o controlados con estrategias y un enfoque preventivo primordial que incluya mejorar la salud materna, paterna e infantil, lo cual permitirá reducir la carga global de HTA y ECV en la vida futura.

\section{REFERENCIAS}

1. Flynn JT, Kaelber DC, Baker-Smith CM, Blowey D, et al. Clinical Practice Guidelines for Screening and Management of Hight Blood Pressure in Children and Adolescents. Pediatrics. 2017;140(3):e2017904.

2. Lurbe E, Agabiti-Roseic E, Kennedy Cruickshankd J, Dominiczaket A, et al. 2016 European Society of Hypertension Guidelines for the Management of high blood pressure in children and adolescents. J Hypertens. 2016 Oct;34(10): 1887-1920.

3. Sinha MD. From Pregnancy to Childhood and Adulthood: The Trajectory of Hypertension. In: Lurbe E, Whül E (eds). Hypertension in children and adolescent: New perspectives. Cham: Springer; 2019.Págs.1-16.

4. IngelfingerJR. The Contributions of Perinatal Programming to Blood Pressure Levels in Childhood and Beyon. In: Lurbe E, Whül E (eds). Hypertension in children and adolescent: New perspectives. Cham: Springer; 2019.Págs.17-30.

5. Singh RR, Denton KM, Bertram JF. Perinatal Programming of Arterial Pressure. In: Flynn JT, Ingelfinger J, Redwine K(eds). Pediatric Hypertension. 4th ed. Cham: Springer; 2018.Págs.135-58.

6. Ingelfinger J, Nuyt AM. Impact of fetal programming, birth weight, and infant feeding on later hypertension. $J$ Clin Hypertens (Greenwich). 2012; 14(6):365-71.

7. Ingelfinger JR, Woods LL. Perinatal programming, renal development, and adult renal function. Am J Hypertens. 2002; 15(2 Pt 2):S46ロ9.

8. Nuyt AM. Mechanisms underlying developmental programming of elevated blood pressure and vascular dysfunction: evidence from human studies and experimental animal models. Clin Sci (Lond). 2008;114(1):117.

9. Poston L. Influence of maternal nutritional status on vascular function in the offspring. Microcirculation. 2011; 18(4):256-62.

10. Barker DJ, Osmond C. Death rates from stroke in England and Wales predicted from past maternal mortality. $\mathrm{Br} \mathrm{Med}$ J (Clin Res Ed). 1987; 295(6590):83-6.

11. Barker DJ, Bull AR, Osmond C, Simmonds SJ. Fetal and placental size and risk of hypertension in adult life. BMJ. 1990; 301(6746):259-62.

12. Roseboom TJ, van der Meulen JH, Osmond C, Barker DJ, et al. Coronary heart disease after prenatal exposure to the Dutch famine, 1944-45. Heart. 2000; 84(6):595-8.
13. Stein AD, Zybert PA, van der Pal-de Bruin K, Lumey LH. Exposure to famine during gestation, size at birth, and blood pressure at age $59 \mathrm{y}$ : evidence from the Dutch famine. Eur J Epidemiol. 2006; 21(10):759-65.

14. Eriksson JG, Forsén TJ, Kajantie E, Osmond C, Barker DJ. Childhood growth and hypertension in later life. Hypertension. 2007; 49(6):1415-21.

15. de Jong F, Monuteaux MC, van Elburg RM, Gillman MW, BelfortMB.Systematic review and meta-analysis of preterm birth and later systolic blood pressure. Hypertension. 2012; 59(2):226-34.

16. Adair LS, Cole TJ. Rapid child growth raises blood pressure in adolescent boys who were thin at birth. Hypertension. 2003; 41(3):451-6.

17. Barker DJ, Osmond C, Forsén TJ, Kajantie E, Eriksson JG. Trajectories of growth among children who have coronary events as adults. N Engl J Med. 2005; 353(17):1802-9.

18. Ferraro AA, Barbieri MA, da Silva AAM, Grandi C, et al. Contributions of relative linear growth and adiposity accretion from birth to adulthood to adult hypertension. Sci Rep. 2017; 7(1):8928.

19. Parkinson JR, Hyde MJ, Gale C, Santhakumaran S, Modi N. Preterm birth and the metabolic syndrome in adult life: a systematic review and meta-analysis. Pediatrics. 2013; 131(4):e1240-63.

20. Brennan KA, Olson DM, Symonds ME. Maternal nutrient restriction alters renal development and blood pressure regulation of the offspring. Proc Nutr Soc. 2006; 65(1):11624.

21. King JC. Maternal obesity, metabolism, and pregnancy outcomes. Annu Rev Nutr. 2006; 26:271-91.

22. Chourdakis E, Fouzas S, Papadopoulou C, Oikonomou N, et al. Effect of Early-Onset Preeclampsia on Offspring's Blood Pressure during the First Month of Life. J Pediatr. 2020; 220:21-6.e1.

23. Dasinger JH, Alexander BT. Gender differences in developmental programming of cardiovascular diseases. Clin Sci (Lond). 2016; 130(5):337-48.

24. Blake KV, Gurrin LC, Evans SF, Beilin LJ, et al. Maternal cigarette smoking during pregnancy, low birth weight and subsequent blood pressure in early childhood. Early Hum Dev. 2000; 57(2):137-47.

25. Kenna K, Sozo F, De Matteo R, Hanita T, et al. Alcohol exposure during late gestation: multiple developmental outcomes in sheep. J Dev Orig Health Dis. 2012;3(4):224-36.

26. Thomal JT, Palma BD, Ponzio BF, Franco MCP, et al. Sleep restriction during pregnancy: hypertension and renal abnormalities in young offspring rats. Sleep. 2010; 33(10):1357-62.

27. McGowanPO,MatthewsSG.Prenatalstress, glucocorticoids, and developmental programming of the stress response. Endocrinology. 2018; 159(1):69-82.

28. Anwar MA, Saleh AI, Al Olabi R, Al Shehabi TS, Eid AH. Glucocorticoid-induced fetal origins of adult hypertension: association with epigenetic events. Vasc Pharmacol. 2016; 82:41-50.

29. Cottrell EC, Seckl JR. Prenatal stress, glucocorticoids and the programming of adult disease. Front Behav Neurosci. 2009; 3:19.

30. Stark MJ, Wright IM, Clifton VL. Sex-specific alterations in placental 11beta-hydroxysteroid dehydrogenase 2 activity and early postnatal clinical course following antenatal betamethasone. Am J Physiol Regul Integr Comp Physiol. 2009; 297(2):R510-4.

31. Christian P, StewartCP. Maternal micronutrient deficiency, fetal development, and the risk of chronic disease. J Nutr. 2010; 140(3):437-45.

32. Arredondo BA, Guerrero JG, Arredondo RA. Relationship 
between intestinal microbiota and blood pressure. MediSan. 2019; 23(5):967-80.

33. Taal HR, Geelhoed JJ, Steegers EA, Hofman A, et al. Maternal smoking during pregnancy and kidney volume in the offspring: the Generation R Study. Pediatr Nephrol. 2011; 26(8):1275-83.

34. Gray SP, Denton KM, Cullen-McEwen L, Bertram JF, Moritz K. Prenatal exposure to alcohol reduces nephron number and raises blood pressure in progeny. J Am Soc Nephrol. 2010; 21(11):1891-902.

35. Das SK, McIntyre HD, Alati R, Al Mamun A. Maternal alcohol consumption during pregnancy and its association with offspring renal function at 30 years: observation from a birth cohort study. Nephrology (Carlton). 2019; 24(1):21-7.

36. Harskamp-van Ginkel MW, Ierodiakonou D, Margetaki K, VafeiadiM, etal.Gestational sleep deprivation is associated with higher offspring body mass index and blood pressure. Sleep. 2020; 43(12):zsaa110.

37. Gutiérrez Arzapalo PY. Alteraciones estructurales y mecánicas vasculares asociadas a la programación fetal de lahipertensión arterial. Tesis doctoral.Madrid:Universidad Autónoma de Madrid; 2016.

38. Luyckx VA, Bertram JF, Brenner BM, Fall C, et al. Effect of fetal and child health on kidney development and longterm risk of hypertension and kidney disease. Lancet. 2013; 382(9888):273-83.

39. Brenner BM, Chertow GM. Congenital oligonephropathy and the etiology of adult hypertension and progressive renal injury. Am J Kidney Dis. 1994; 23(2):171 $\square 5$.

40. Mañalich R, Reyes L, Herrera M, Melendi C, Fundora I. Relationship between weight at birth and the number and size of renal glomeruli in humans: a histomorphometric study. Kidney Int. 2000; 58(2):770-3.

41. Denic A, Lieske JC, Chakkera H.A, Poggio ED, et al. The Substantial Loss of Nephrons in Healthy Human Kidneys with Aging. J Am Soc Nephrol. 2017; 28(1):313-20.

42. Cheng CJ, Lozano G, Baum M. Prenatal programming of rat cortical collecting tubule sodium transport. Am J Physiol Renal Physiol. 2012; 302(6):F674-8.

43. Wesseling S, Koeners MP, Joles JA. Salt sensitivity of blood pressure: developmental and sex-related effects. Am J Clin Nutr. 2011; 94(6 Suppl):S1928-32.

44. Alexander BT, Hendon AE, Ferril G, Dwyer TM. Renal denervation abolishes hypertension in low-birth-weight offspring from pregnant rats with reduced uterine perfusion. Hypertension. 2005; 45(2):754-8.

45. Amaral MS, Ribeiro CC, Alves MT, Ribeiro MJ, et al. Modeling Pathways From the Perinatal Factors to the Vascular Risk Phenotype at the End of the Second Decade of Life: Birth Cohort, Brazil. Hypertension. 2020; 76(2):35965.

46. Wang KC, Botting KJ, Padhee M, Zhang S, et al. Early origins of heart disease: low birth weight and the role of the insulin-like growth factor system in cardiac hypertrophy. Clin Exp Pharmacol Physiol. 2012; 39(11):958-64.

47. Zohdi V, Lim K, Pearson JT, Black MJ. Developmental programming of cardiovascular disease following intrauterine growth restriction: findings utilising a rat model of maternal protein restriction. Nutrients. 2014; 7(1):119-52.

48. Gezmish O, Tare M, Parkington HC, Morley R, et al. Maternal vitamin D deficiency leads to cardiachypertrophy in rat offspring. Reprod Sci. 2010; 17(2):168-76.

49. Morrison JL, Botting KJ, Dyer JL, Williams SJ, et al. Restriction of placental function alters heart development in the sheep fetus. Am J Physiol Regul Integr Comp Physiol. 2007; 293(1):R306-13.

50. Lewandowski AJ, Levy PT, Bates ML, McNamara PJ, et al.
Impact of the vulnerable preterm hearth and circulation on adult cardiovascular disease risk. Hypertension. 2020; 76(4):1028-37.

51. Jouret B, Dulac Y, Bassil Eter R, Taktak A, et al. Endothelial functional and mechanical arterial properties in children born small for gestational age: comparison with obese children. Horm Res Pediatr. 2011; 76(4):240-7.

52. Martin H, Hu J, Gennser G, Norman M. Impaired endothelial function and increased carotid stiffness in 9-year-old children with low birthweight. Circulation. 2000; 102(22):2739-44

53. Raffetto JD, Khalil RA. Matrix metalloproteinases and their inhibitors in vascular remodeling and vascular disease. Biochem Pharmacol. 2008; 75(2):346-59.

54. Guarner-Lans V, Ramírez-Higuera A, Rubio-Ruiz ME, Castrejón-Téllez V, et al. Early Programming of Adult Systemic Essential Hypertension. Int J Mol Sci. 2020; 21(4):1203.

55. Martyn CN, Greenwald SE. A hypothesis about a mechanism for the programming of blood pressure and vascular disease in early life. Clin Exp Pharmacol Physiol. 2001; 28(11):948-51.

56. Cheung YF, Wong KY, Lam BC, Tsoi NS. Relation of arterial stiffness with gestational age and birth weight. Arch Dis Child. 2004; 89(3):217-21.

57. Nilsson PM, Lurbe E, Laurent S. The early life origins of vascular ageing and cardiovascular risk: the EVA syndrome. J Hypertens. 2008; 26(6):1049-57.

58. Grassi G, Mark A, Esler M. The sympathetic nervous system alterations in human hypertension. Circ Res. 2015; 116(6):976-90.

59. Wyss JM. The role of the sympathetic nervous system in hypertension. Curr Opin Nephrol Hypertens. 1993; 2(2):26573.

60. Norlander AE, Madhur MS, Harrison DG. Theimmunology of hypertension. J Exp Med. 2018; 215(1):21-33.

61. Su Y, Bi J, Pulgar VM, Figueroa J, et al. Antenatal glucocorticoid treatment alters $\mathrm{Na}+$ uptake in renal proximal tubule cells from adult offspring in a sex-specific manner. Am J Physiol Renal Physiol. 2015; 308(11):F1268-75.

62. Tain YL, Lin YJ, Sheen JM, Yu HR, et al. High Fat Diets SexSpecifically Affect the Renal Transcriptome and Program Obesity, Kidney Injury, and Hypertension in the Offspring. Nutrients. 2017; 9(4):357.

63. Woods LL, Ingelfinger JR, Nyengaard JR, Rasch R. Maternal protein restriction suppresses the newborn reninangiotensin system and programs adult hypertension in rats. Pediatr Res. 2001; 49(4):460-7.

64. Wu L, Shi A, Zhu D, Bo L, et al. High sucrose intake during gestation increases angiotensin II type 1 receptor-mediated vascular contractility associated with epigenetic alterations in aged offspring rats. Peptides. 2016; 86:133-44.

65. Moritz KM, Cuffe JS, Wilson LB, Dickinson H, et al. Review: sex specific programming: a critical role for the renal reninangiotensin system. Placenta. 2010; 31 (Suppl):S40-6.

66. Liang M. Epigenetic Mechanisms and Hypertension. Hypertension. 2018; 72(6):1244-54.

67. Tollefsbol TO. Epigenetics: The New Science of Genetics. In: Tollefsbol TO (ed). Handbook of Epigenetics: The New Molecular and Medical Genetics. Amsterdam: Academic Press; 2011:1-6.

68. Stoll S, Wang Ch, Qiu H. DNA Methylation and Histone Modification in Hypertension. Int JMol Sci. 2018;19(4):1174.

69. Wang J, Gong L, Tan Y, Hui R, Wang Y. Hypertensive epigenetics: from DNA methylation to microRNAs. J Hum Hypertens. 2015; 29(10):575-82.

70. Mendizábal B, Urbina E, Becker R, Daniels SR, et al. SHIPAHOY (Study of High Blood Pressure in Pediatrics: Adult 
Hypertension Onset in Youth): rationale, design, and methods. Hypertension. 2018; 72(3):625-31.

71. Heijmans BT, Tobi EW, Stein AD, Putter H, et al. Persistent epigenetic differences associated with prenatal exposure to famine in humans. Proc Natl Acad Sci U S A. 2008; 105(44):17046-9.

72. Tobi EW, Lumey LH, Talens RP, Kremer D, et al. DNA methylation differences after exposure to prenatal famine are common and timing- and sex-specific. Hum Mol Genet. 2009; 18(21):4046-53.

73. Sinclair KD, Allegrucci C, Singh R, Gardner DS, et al. DNA methylation, insulin resistance, and blood pressure in offspring determined by maternal periconceptional B vitamin and methionine status. Proc Natl Acad Sci U S A. 2007; 104(49):19351-6.

74. Ramírez R. Programación fetal de la hipertensión arterial del adulto: mecanismos celulares y moleculares. Rev Colomb Cardiol. 2013; 20(1):23-32.

75. Bygren LO, Kaati G, Edvinsson S. Longevity determined by paternal ancestors' nutrition during their slow growth period. Acta Biotheor. 2001; 49(1):53-9.
76. Hallows SE, Regnault TR, Betts DH. The long and short of it: the role of telomeres in fetal origins of adult disease. J Pregnancy. 2012; 2012:638476.

77. Thompson LP, Al-Hasan Y. Impact of oxidative stress in fetal programming. J Pregnancy. 2012; 2012:582748.

78. Franco MC, Akamine EH, Rebouças N, Carvalho MH, et al. Long-term effects of intrauterine malnutrition on vascular function in female offspring: implications of oxidative stress. Life Sci. 2007; 80(8):709-15.

79. Ávila JG, Echeverri I, de Plata CA, Castillo A. Impact of oxidative stress during pregnancy on fetal epigenetic patterns and early origin of vascular diseases. Nutr Rev. 2015; 73(1):12-21.

80. Falkner B, Lurbe E. Primordial Prevention of High Blood Pressure in Childhood: an opportunity not to be missed. Hipertension. 2020; 75(5):1142-50.

81. Luyckx VA, Perico N, Somaschini M, Manfellotto D, et al. A developmental approach to the prevention of hypertension and kidney disease: a report from the Low Birth Weight and Nephron Number Working Group. Lancet. 2017; 390(10092):424-8. 